\title{
Effects of stochasticity on the length and behaviour of ecological transients
}

\author{
Alan Hastings, ${ }^{1 *, 2}$ Karen C. Abbott,${ }^{3}$ Kim Cuddington, ${ }^{4}$ Tessa Francis, ${ }^{5}$ \\ Ying-Cheng Lai, ${ }^{6}$ Andrew Morozov, ${ }^{7,8}$ Sergei Petrovskii, ${ }^{7}$ Mary Lou Zeeman ${ }^{9}$ \\ ${ }^{1}$ Department of Environmental Science and Policy, \\ University of California, Davis, CA 95616, USA \\ ${ }^{2}$ Santa Fe Institute, 1399 Hyde Park Road, Santa Fe, NM 87501, USA \\ ${ }^{3}$ Department of Biology, Case Western Reserve University, Cleveland, OH 44106, USA \\ ${ }^{4}$ Department of Biology, University of Waterloo, Waterloo, Ontario, N2L 3G1, Canada \\ ${ }^{5}$ Puget Sound Institute, University of Washington Tacoma, Tacoma, WA 98421, USA \\ ${ }^{6}$ School of Electrical, Computer and Energy Engineering, \\ Arizona State University, Tempe, AZ 85287, USA \\ ${ }^{7}$ School of Mathematics and Actuarial Science, \\ University of Leicester, Leicester LE1 7RH, UK \\ ${ }^{8}$ Institute of Ecology and Evolution, Russian Academy of Sciences, Moscow, Russia \\ ${ }^{9}$ Department of Mathematics, Bowdoin College, Brunswick, ME 04011, USA
}

*To whom correspondence should be addressed; E-mail: amhastings@ucdavis.edu

March 25, 2021 


\begin{abstract}
There is a growing recognition that ecological systems can spend extended periods of time far away from an asymptotic state, and that ecological understanding will therefore require a deeper appreciation for how long ecological transients arise. Recent work has defined classes of deterministic mechanisms that can lead to long transients. Given the ubiquity of stochasticity in ecological systems, a similar systematic treatment of transients that includes the influence of stochasticity is important. Stochasticity can of course promote the appearance of transient dynamics by preventing systems from settling permanently near their asymptotic state, but stochasticity also interacts with deterministic features to create qualitatively new dynamics. As such, stochasticity may shorten, extend, or fundamentally change a system's transient dynamics. Here, we describe a general framework that is developing for understanding the range of possible outcomes when random processes impact the dynamics of ecological systems over realistic time scales. We emphasize that we can understand the ways in which stochasticity can either extend or reduce the lifetime of transients by studying the interactions between the stochastic and deterministic processes present, and we summarize both the current state of knowledge and avenues for future advances.
\end{abstract}




\section{Introduction}

Two major goals of ecological theory are to make predictions, and to explain past observations. In both cases, qualitative changes in dynamics through time represent both a challenge and an opportunity. For prediction, a sudden change in dynamics is important to capture. In parallel, understanding the limits to prediction, in time or in other ways, is important. Both for prediction of the future and for understanding the processes that lead to the current state of the system, the presence of large changes in dynamics, sometimes referred to as black swan events $[1,2]$, presents a challenge. How can these events be understood using ecological models?

There is increasing recognition that transients can play a critical role in ecological systems $[3,4,5,6,7,8,9,10]$, building on the variety of long transient behaviors exhibited by nonlinear dynamical systems $[11,12]$. For example, regime shifts are an important phenomenon in ecology, in which the system behavior changes suddenly without any warning (e.g., sudden species extinction) $[13,14,15]$. The traditional view is that regime shifts are caused by parameter drifting. However, as recently emphasized, even without any parameter change, transient behaviour can lead to regime shifts $[8,9]$.

Earlier work has emphasized the possibility of sudden changes in dynamics even in deterministic models with constant parameters [8], but stochasticity is ubiquitous in real ecosystems and will affect transients [10]. How has the deterministic view limited our understanding of sudden shifts in ecosystems, and how does this understanding deepen when we account for stochasticity in our theoretical constructs and models? This question, in the context of observations of changing ecological dynamics and black swan events in ecology [1, 2], fits in with the recent recognition of the importance of focusing on dynamics on ecological time scales. Stochasticity can play an important role in determining dynamics on realistic time scales.

Real-world ecosystems are subject to inevitable and constant influences of stochastic disturbances that can have significant effects on the population dynamics $[16,17,18,19,20,21,22,23$, $24,25,26,27,28,29,30,8]$. A particularly notable example includes the population dynamics of Dungeness crab, Cancer magister, along the USA West Coast [31]. In this system, chaoticlike oscillations were analyzed using a method that combined data analysis and modelling fitted from data to reveal that the oscillations were actually long transient relaxations due to stochastic perturbations of a stable equilibrium. In addition, random perturbations of cyclic population dynamics can also result in a chaotic-like behaviour, which was observed in the experimental dynamics of Tribolium [32]. Although there have been many individual, well-studied examples illustrating these points, a recognition of common themes arising in a discussion of stochastic transients in ecological systems reveals both new insights into ecological dynamics and suggests important future research directions.

We start from the premise that in natural systems, noise and random disturbances are inevitable. We consider noise that affects one or more state variables, perturbing them with some magnitude, direction, and frequency. Noise can influence long transients in a variety of ways (figure 1). Noise may certainly alter long transient dynamics that were created by another mechanism and already present in the ecological system. Importantly, stochasticity can also provide an alternate mechanism for long transient dynamics, creating a long transient that would not otherwise occur. There are two major types of stochasticity in ecological systems: external perturbations due to random variations in the environmental conditions, and internal population fluctuations. Some environmental stochasticity can be modeled as additive Gaussian white noise [33, 34], while internal stochasticity is effectively demographic noise [35, 23, 36, 37] that needs to be described as multiplicative noise with its strength depending on the fluctuating abundance variable. Demographic noises are thus correlated, colored stochastic processes.

We begin this exploration of the role of noise in creating and influencing long transients 


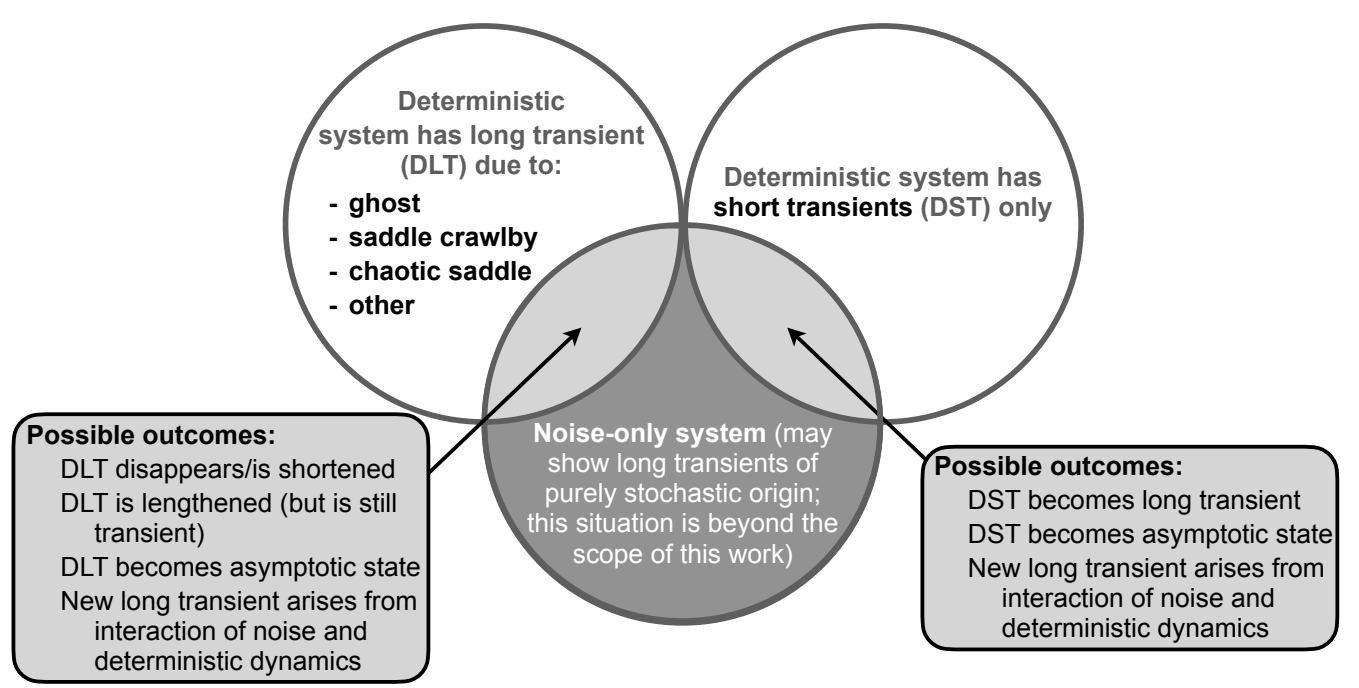

Figure 1: Real world dynamics fall into the light gray regions, where deterministic and stochastic processes interact. The length and nature of transient dynamics in these regions depends both on the presence of deterministic features known to promote long transients and on properties of the noise. $\mathrm{DLT}=$ deterministic long transient (i.e. a long transient that exists in the deterministic part of the dynamics); DST = deterministic short transient. The definition of a 'long' transient can be found in Section 3, and for simplicity we refer to all other transients as 'short'.

with some simple examples, illustrating the important point that stochasticity can either extend or reduce transients. Using the simple examples as a jumping off point, we then undertake a systematic exploration of transients in nonlinear (density-dependent) ecological systems. Even these simple examples bring out the important point that the definition of a transient for a stochastic system may be less clear cut than for a deterministic one. In particular, we have to be clear about terminology for the case where there is no long transient for the deterministic skeleton of a model, yet the addition of stochasticity produces long term dynamics that are different than the equilibrium dynamics of the underlying deterministic model. As a way to outline the framework of the current paper, we summarize the current state of synthesis in Figure 1. The more systematic approach suggested by this figure first requires attention to the definition of transients and the kinds of stochasticity we consider, followed by different ways in which transients arise and the effects of stochasticity in different cases.

\section{Simple examples of transients}

Before starting with a systematic exploration of the influence of stochasticity on transients with an emphasis on long transients, simpler systems can provide background. Starting with the simplest case of linear deterministic systems, and then adding stochasticity, will demonstrate first the ecological importance of the phenomena, and provide insights into the role of stochasticity.

Age structured systems provide some of the simplest examples of transient dynamics, which are present even in linear systems. The dynamics of a population of salmon provide a straightforward illustration [38]. Individuals of most salmon species typically reproduce once and then die. In addition, in many populations, almost all individuals reproduce at the same age. We can denote the number of females of age $i$ at time $t$ by $n_{i}(t)$ in a discrete time description. We assume that the survival from age 1 to age 2 is given by $s_{1}$ and similarly by $s_{2}$ for age 2 to 3. Finally, denote the fecundity of 2-year-olds by $m_{2}$ with $m_{2}>1$ and the fecundity of 1 - and 
3 -year-olds by $\epsilon_{1}$ and $\epsilon_{3}$ respectively, where $\epsilon_{i} \ll 1$. Thus the dynamics of the females would be given by the following Leslie matrix model, if almost all individuals reproduce at age 2 :

$$
\left(\begin{array}{l}
n_{1}(t+1) \\
n_{2}(t+1) \\
n_{3}(t+1)
\end{array}\right)=\left(\begin{array}{ccc}
\epsilon_{1} & m_{2} & \epsilon_{3} \\
s_{1} & 0 & 0 \\
0 & s_{2} & 0
\end{array}\right)\left(\begin{array}{l}
n_{1}(t) \\
n_{2}(t) \\
n_{3}(t)
\end{array}\right) .
$$

It is easy to see that if $\epsilon_{1}=\epsilon_{3}=0$, this matrix would have two dominant eigenvalues of the same magnitude. If instead these fecundities are small and positive, then these two eigenvalues would have nearly the same magnitude. In this case, if in a given year almost all individuals were of age 2 and very few were of age 1, then for many years the dominant age class would alternate between 1 and 2 . The addition of stochasticity could greatly reduce the time the system would need to approach stable age distribution (i.e. where the ratio of individuals in different age classes would be constant from year to year).

A second example of a linear ecological transient is given by a simple predator-prey system with an equilibrium that is a stable focus, but with complex eigenvalues with very small, negative real parts. In this case, the deterministic system would have oscillations whose magnitude would decay very slowly, while the presence of environmental stochasticity could extend the time to reach equilibrium [39] by interrupting the decay in cycle magnitudes.

What is interesting about these two simple examples is the contrasting effect of stochasticity. In the first one, the Leslie matrix model, clearly stochasticity would shorten the transient by accelerating the approach to the stable age distribution. In contrast, for the predator prey models, as originally demonstrated $[40,39]$ using Fourier analysis, a stochastic system can continue to exhibit cyclic behavior indefinitely and thus stochasticity greatly extends the lifetime of the transient, even making it effectively infinite.

If even linear systems can exhibit interesting and contrasting effects of stochasticity on transients, nonlinear systems, which can exhibit longer and more varied kinds of transients [8], will provide a much richer set of phenomena that will be key for ecological understanding. But, before presenting a systematic exploration of long nonlinear transients, it is important to highlight a particular class of stochastic transients which are prominent in ecology and more broadly.

Increasing attention is being paid to tipping points, and early warning signs for tipping have been developed based on the concept of critical slowing down [41, 42]. Critical slowing down describes the time a system takes to respond to a perturbation. Without stochasticity to perturb a system, there would be no opportunity to observe critical slowing down, and thus no possibility of early warning signs. Thus, the concept of critical slowing down has at its core ideas about both transients and stochasticity.

Transients also enter into another important aspect of early warnings. An emphasis of early warning sign work has been the detection of parameter changes that push the system through a saddle node bifurcation. At such a bifurcation, the system's stable equilibrium is replaced by a ghost attractor which can lead to a long transient [7]. A natural question is how stochasticity affects the length (in time) of a transient resulting from a ghost attractor.

These simple but illustrative examples provide an important starting point for a discussion of transients in stochastic systems. But transients arise in many other ways, and given the ubiquity of stochasticity, a more thorough and systematic investigation is called for. Clearly, first steps are an unambiguous definition of transients, and a consideration of how stochasticity enters into ecological systems. 


\section{Definitions of long transients}

There are two different ways to define transients in mathematical models (including those with noise) as well as in empirical systems. In this study we are emphasizing long transients due to their crucial role in ecological applications including sudden regime shifts.

Consider first the scenario where the system is functioning in a certain dynamical regime in which its major characteristics remain unchanged for a long time (for stochastic systems we operate with average characteristics). Here by 'long time' we understand the situation where the duration of the regime is much longer that its internal characteristic time (e.g. the period of oscillations). In an ecological context, this signifies that the duration of the regime is much longer than the generation time of species involved. To the external observer exploring the system based on time series, such a system would appear to be stable. Now suppose that at some moment in time, but without any changes to the properties governing the dynamics, the system demonstrates a rapid transition (as compared to the duration of the regime) to another regime which, in turn, conserves its new characteristics unchanged for a long time again. In this case, we call the preceding dynamical regime a long transient. Note that the post-transitional regime can be transient as well and a new transition may occur later on. In fact, the above mentioned scenario of transient behaviour describes a shift between regimes. It is also important to emphasize that according to the considered scenario the transition between regimes occurs without external forcing of the system, i.e. without changing model parameters in the course of time. Obviously, however, the presence of the long transient depends on the initial conditions for the system, so a regime shift due to long transients may be originally triggered by some initial disturbance of either the parameters or state of the system.

The other long transient scenario involves the situation where the system itself is in slow transition to a stable or quasi-stable state. We assume that the pattern of dynamics evolves very slowly with time as compared to the characteristic time of the current system. For example, this can be the case of damped oscillations with a very long relaxation time where both the amplitude and the period change only slightly. An important practical case is where the transition of the system to the final attractor actually requires an arbitrarily large time [31]. This can happen in the presence of large noise since there will always be perturbations kicking the system away from the eventual asymptotic state. In this case, the resultant pattern of dynamics will be an infinite sequence of transient regimes. We note that calling this behavior a transient is, perhaps, an arbitrary decision, as the combination of stochasticity plus the deterministic skeleton produces behavior that persists indefinitely. We believe that this is the more useful choice because it encompasses the role that stochasticity plays in altering dynamics and also note that this provides consistency in our definition.

Earlier [9] a key property of long transients was described: there is a scaling law describing the duration of transients while a particular model parameter is varied. The length of a transient regime (in stochastic systems the length should be understood as the mean length) can be made as large as possible when a certain bifurcation parameter (including the magnitude of noise) approaches a critical value. This mathematically quantifies the common sense notion of 'long' transient (i.e. how long is long). From the ecological point of view, the duration of a transient is always limited by natural constraints and we usually assume the average length of transients to be larger than several characteristic generation times [8]. The existence of a scaling law allows us to classify transients into different types [9]. 


\section{Types of noise}

In this contribution we focus primarily on extrinsic temporal noise, that is, those sources of stochasticity that arise because of relationships and quantities external to the modelled system. The classic example is, of course, detrended environmental variation. Coulson et al. [43] describe active and passive stochasticity, where active noise interacts with deterministic nonlinearity to produce dynamics that cannot result from either factor independently [44], and passive noise influences the transients among different deterministic states. The impact of environmental noise that affects the modelled system will depend on the modelled time frame of interest, the influence of the particular factor, the time scales of variation in the noise relative to the time scales of response, and the characteristics of the noise itself.

There are many properties to consider such as the whether the stochasticity is continuous, a single perturbation or seasonal, whether it has larger or smaller magnitude, and whether it has frequencies in a similar range as the intrinsic dynamics. Understanding the structure of noise is critical for understanding its potential impact. For example, continuous long term trended variation such as climate change, short term uncorrelated variation, and directed impacts through management might all be expected to have different effects on the same system.

The most familiar description of environmental stochasticity is as random draws, independent in time, from a Guassian distribution with small variance. In this white noise process, deviations from the mean at one timestep are unrelated to the size and magnitude of deviations at another timestep. That is, white noise is uncorrelated in time. Or put another way, all frequency components of the signal have the same expected value. The fact that the variance of noise is small has little bearing on its dynamical impact. To take a trivial case, even small variations close to a critical value in a bifurcation parameter can have large impact on the dynamics of a system. Environmental stochasticity of relatively small variance can also create oscillations through resonance effects $[39,45]$.

Of course most environmental signals such as temperature, rainfall and river flow rates have large variance and are autocorrelated in time even after detrending (e.g., [46, 47]). The strength of this autocorrelation depends on the signal itself (e.g., air temperature vs. sea surface temperature), the geographic location (e.g., continental air temperatures vs maritime air temperatures), and the time period. In particular climate change is altering the autocorrelation of related environmental signals $[48,49]$. The autocorrelation of deviations can be large, and, in these cases, can cause clustering of extreme events [50]. Therefore, when we model the impact of environmental stochasticity as a white noise process, we may err in our estimates of the probability of long transient behaviour, as these signals can push a system away from (or towards) an attractor by virtue of the autocorrelated variation.

Of course larger amplitude noise, for example seasonal forcing [51, 52], can have large impacts, while episodic large amplitude noise (flow-kick) can move and maintain a system far from any attractor in the deterministic scaffold [53].

\section{$5 \quad$ Interactions between stochasticity and transients}

The effects of noise on transients are numerous and diverse (Fig. 1). Noise can make the lifespan of the transient considerably shorter and/or decrease the range of the initial conditions that result in the long transient dynamics, or remove the long transient altogether. Alternatively, noise can make the transient's lifespan longer. With noise, the emergence of long transient dynamics becomes a probabilistic event rather than a deterministic one. Noise can turn a deterministic long transient into stable, persistent dynamics [40, 39]. Moreover, noise can create 
long transients via mechanisms that do not exist in a deterministic case $[54,55,56]$.

The outcome of the interaction between noise and a long transient depends both on the properties of noise and on the mechanism behind the (deterministic) long transient. For the transient created by a crawlby [8] (i.e. caused by the closeness of the system to a saddle point), it is readily seen that uncorrelated noise makes the lifespan of the transient shorter (but does not remove it unless the noise is large), as the random movement of the system in the phase space pushes it, on average, away from the equilibrium. Consequently, the system does not necessarily follow the phase flow along the stable manifold that otherwise would bring it into the close vicinity of the saddle (cf. Fig. 2 in [9]). Interestingly, in the presence of noise, long transient dynamics can also emerge, with a certain probability, for a set of initial conditions that would not otherwise lead to a long transient, as the random movement of the system in the phase space can occasionally bring the system into close vicinity of the saddle.

The effect of correlated, directed noise can also make a transient much longer, by keeping the system in the vicinity of a saddle or ghost attractor. In particular, this is readily seen in a flow-kick system [57, 53] where the kicks (directed, quasi-periodical, time-discrete random perturbations of the state variable) control the movement of the system over the phase space,

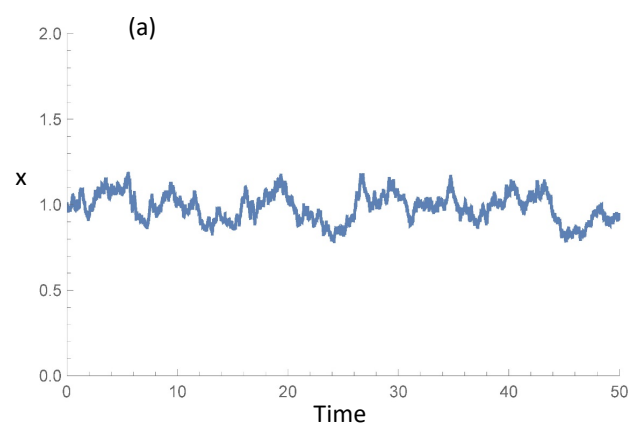

(c)

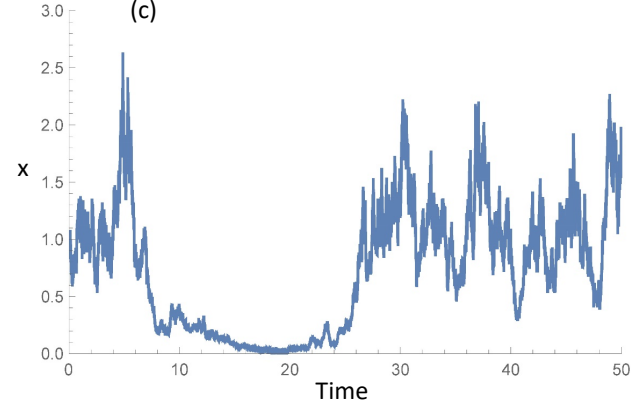

(b)

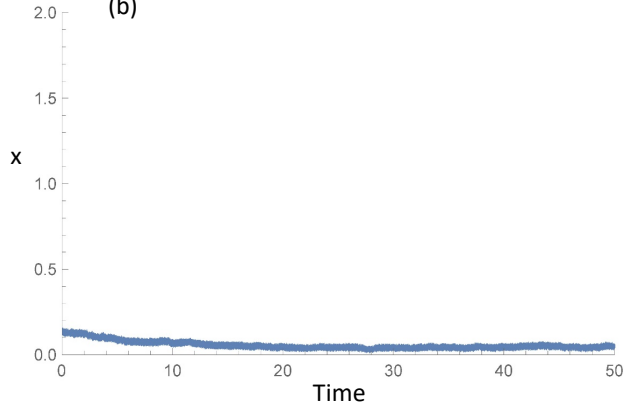

(d)

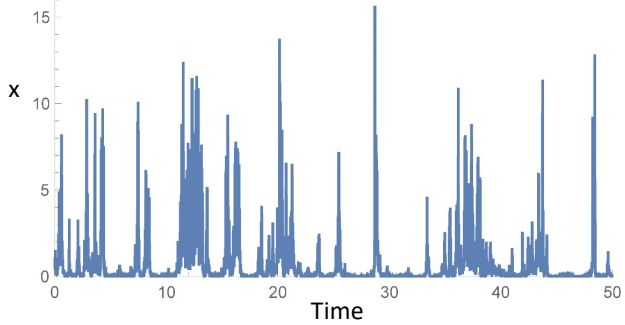

Figure 2: Behavior of a bistable ecological model with different noise levels. In each case the model with the deterministic skeleton $d x / d t=x(x-0.3)(1-x)+0.01$, describing the dynamics of a population with scaled population size $x$ describing Allee dynamics, is simulated for different noise levels. The deterministic skeleton is a bistable system where the last term represents a small steady immigration to prevent extinction. The noise term is of the form $\gamma x w$ where $w$ is a Wiener process (white noise) with mean 0 and variance 1 and the equation is integrated as the equivalent Stratanovich stochastic differential equation using an Euler method and step size of 0.001. Note scale differences. (a) Small noise level, $\gamma=0.1$, starting near the larger equilibrium; system stays near the equilibrium; (b) small noise level, $\gamma=0.1$, starting near the lower equilibrium; system stays near the equilibrium; (c) intermediate noise level, $\gamma=0.6$, showing noise induced transients; and (d) large noise level, $\gamma=5$, where the system is noise dominated and does not exhibit transients. 
with the capacity of keeping it close to a specific location in that space (e.g. a saddle or a ghost attractor).

Perhaps the simplest and best known example where noise can change the system properties qualitatively is the bistable system. We mention here that bistable systems are highly ecologically relevant; in particular, they are used as the paradigm of a regime shift [58] resulting from slow parameter change. Without noise or even with extremely small noise, the system remains in the vicinity of one of the steady states indefinitely long (see Fig. 2a,b). However, slightly larger uncorrelated noise can push the system out of the attraction basin of the current state, so that it fast converges to the alternative state: a purely noise-induced regime shift occurs. The dependence of the state variable (e.g. the population size) on time takes the form of alternating periods with a quasi-stationary value (Fig. 2c). The time spent by the system in the vicinity of the given state is inversely proportionate to the noise intensity and, hence, can be very long. Therefore, small noise creates long transient dynamics. We mention here that there are empirical examples of stochastic switching with long transients in ecological systems [59,60,61] as well as in epidemiology [62]. Interestingly, noise of larger intensity can destroy the long transient as the system diffuses across the whole span of the phase space between the two states (Fig. 2d). Therefore, the dependence of the lifetime of the transient dynamics on the strength of noise is non-monotonous. This non-monotonicity is a generic property of population dynamics with stochasticity and is seen in a variety of systems and models (e.g. see [63, 64, 65]). Below, we see a similar phenomenon emerging in high dimensional ecological systems.

Another example of a situation where noise can create long transients is the dynamics of excitable systems [55]. A relevant ecological system that exhibits excitable dynamics is a preypredator system with Holling type III predation [66]. In a certain parameter range (e.g. where the linear predator nullcline is to the left of the trough of the prey nullcline, see Fig. 3a), the

a

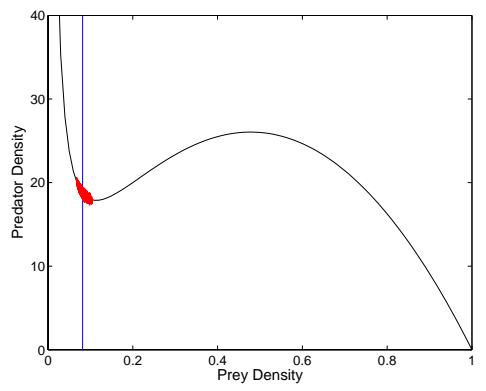

d

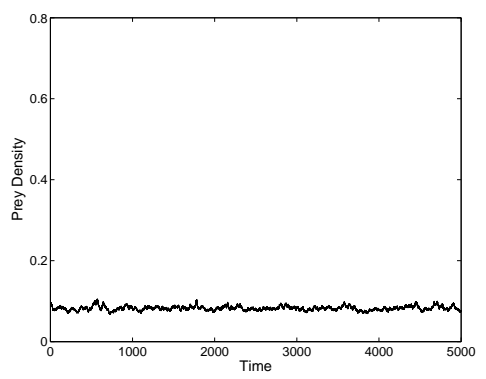

$\mathrm{b}$

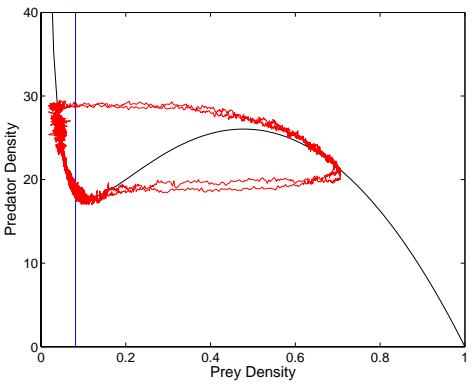

$\mathrm{e}$

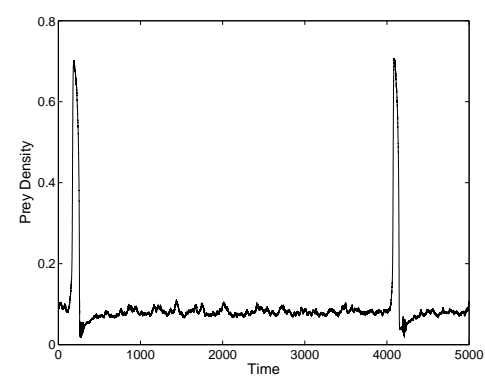

$\mathrm{c}$

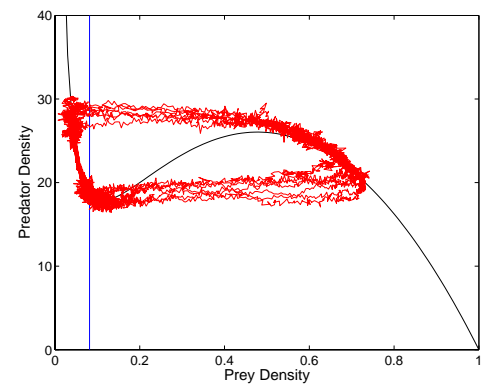

$\mathrm{f}$

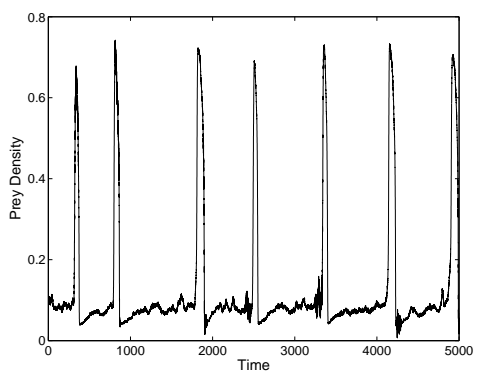

Figure 3: Excitable system under the effect of linear additive noise. (a,b,c) The phase plane of prey-predator system with Holling Type III predation: the irregular red curves show the system trajectories for different level of noise (increasing from a to b to c), the vertical line is the predator nullcline, the S-shaped curve is the prey nullcline. (d,e,f) The corresponding dependence of the prey density on time. 
coexistence state is globally stable, but there is a threshold separating different types of approach to it. For initial conditions on one side of the threshold, the system approaches the steady state directly. For initial conditions on the other, excitable side of the threshold, the system takes an excursion around state space to large abundance of prey and then predator before returning to settle at the steady state. In the deterministic case, once the system has returned to the steady state, it stays there indefinitely. However, the excitability threshold runs close to the steady state, so noise can push the system over the threshold, triggering another large excursion around the phase plane before finally returning to the vicinity of the coexistence state where it can remain for a long time until noise pushes it out again (see Figs. 3b,e). Altogether, the state variable exhibits small-amplitude, random oscillations around the steady state value intermittent with occasional large-amplitude cycles. An increase in the noise level makes the large-amplitude cycles more frequent, see Figs. 3c,f. The periods of small-amplitude oscillations are long transients. This dynamic can be viewed as a noise-induced mixed-mode oscillation $[67,68]$.

Another highly relevant example of transient dynamics facilitated by noise is noise-induced synchronization [56, 69]: population oscillations at different locations in space (e.g. in different patches of a fragmented habitat) that would occur asynchronously in the absence of noise can become synchronized under the effect of noise. In ecology, this phenomenon is often referred to as the Moran effect and it is believed to be responsible for masting [70,71]. However, full synchronization only happens when the controlling parameter (e.g. the strength of the noise) exceeds a certain critical value. In the subcritical parameter range, intermittent synchronization occurs, so that the periods of synchronized and asynchronized dynamics alternate $[72,56]$. In this case, the intervals of synchronized dynamics can be regarded as transients. When the controlling parameter approaches its critical value, their lifetime becomes very long; the average transient time follows the power law [72].

Noise can also turn a transient regime into permanent, sustainable dynamics. As a simple example, let us consider damped population oscillations. In models, such oscillations are frequently observed around a stable focus. Their characteristic life time is $\tau \sim 1 /\left|\operatorname{Re} \lambda_{0}\right|$ where $\lambda_{0}$ is the eigenvalue with the largest negative real part. Correspondingly, for $\left|\operatorname{Re} \lambda_{0}\right| \ll 1$, they last for very long and hence can be regarded as long transient dynamics. The effect of noise can be to turn these long-term damped oscillations into a sustained oscillations $[40,39,73]$ through a mechanism known as stochastic resonance [74]. Such quasi-cycles have been reported in several empirical systems including the dynamics of populations of Dungeness crab [31] and bluefin tuna [75].

As we noted above, tipping points with simple saddle node bifurcations of equilibria are a core example of the interaction between stochasticity and transients. This interaction becomes even more important with a deterministic system with a chaotic attractor that experiences a crisis [76] when its controlling parameter $p$ passes the critical value $p_{c}$. Before the bifurcation point, i.e. for $p<p_{c}$, there is a chaotic attractor so that chaos is self-sustained; at $p=p_{c}$ the chaotic attractor turns into a chaotic saddle so that for $p>p_{c}$ chaotic dynamics are transient. In the presence of chaos, there is a non-zero probability that the system will leave the basin of the attractor; hence the sustainable chaotic dynamics become transient rather than sustained. The classic three species food chain [77] is an important ecological example that has this kind of bifurcation. The presence of noise further complicates the situation and can lead to very long transients, supertransients, even for those parameter values where the deterministic system would have a stable chaotic attractor. As previously reviewed [9], in the region where there is deterministic stability, after some time the corresponding stochastic system can cross the basin boundary and leave the basin of attraction for the chaotic attractor and end up in the basin of attraction for a different attractor. This situation is thus a case where stochasticity leads to a 
transient. A rigorous mathematical analysis of this case is possible [78, 79].

\section{High-dimensional ecological systems}

There is one more important class of systems where stochasticity plays an important role in transients, namely high-dimensional systems. The study of high-dimensional stochastic systems is important because ecological systems are typically high-dimensional, nonlinear and complex. The high dimension can arise either from interactions among many species, or simply from including explicit space in the description. The study of such systems is inherently complex and in its early stages, so rather than give a comprehensive guide, instead we focus on an example that is particular illustrative. A representative class of systems is mutualistic networks [80, $81,82,83,84,85,86,87,88,89,90]$, e.g., a bipartite network of pollinator and plant species. Because the number of species involved in the mutualistic interactions can be large, the system is high dimensional.

Consider a complex mutualistic network subject to environmental or demographic noise, or both. The setting thus naturally has high dimensionality and stochasticity. Can transients arise and are they typical? The answer is affirmative. One scenario is tipping point dynamics [91, 41, $42,92,93,15,94,95,96,97,98,99,83,100,101]$. In particular, in a mutualistic network, the deterministic behavior is dominated by the dynamics about a tipping-point transition $[87,88]$. For example, environmental deterioration will result in massive species extinction, which can occur suddenly in mutualistic systems as a relevant parameter (e.g., the species decay rate) increases through a critical point - a tipping point. Under noise, even when the parameter value has not reached the tipping point, a total system collapse can occur. This is the phenomenon of noise-induced collapse which, dynamically, is nothing but a transition from one steady state to another: from a healthy, high-abundance state to an extinction state. The collapse, of course, does not occur instantaneously: it takes time for the transition to complete, and during this time what we see is a transient. Likewise, when the system is effectively extinct with near zero species abundances, noise can trigger a recovery of the species abundances. In this case, the transition occurs in the opposite direction: from a low abundance steady state to a high abundance one, which is the recently discussed phenomenon of noise-induced recovery [89] accomplished through a transient.

A dynamical picture of the phenomenon of noise-induced collapse and recovery is illustrated in Fig. 4. In the deterministic case, species collapse and recovery are the result of saddlenode bifurcations. Let $\kappa$ be the normalized species decay rate (the bifurcation parameter). Environmental deterioration is manifested as an increase in the value of $\kappa$. As $\kappa$ increases through a critical point, denoted as $\kappa_{c}(0)$, a reverse saddle-node bifurcation occurs, giving rise to a tipping point transition. Now consider the case where noise of amplitude $\varepsilon$ is present. The phenomenon of noise-induced collapse corresponds to an earlier tipping point transition, now occurring at the critical point $\kappa_{c}(\varepsilon)$, where $\kappa_{c}(\varepsilon)<\kappa_{c}(0)$. Likewise, without noise, species recovery occurs through a forward saddle-node bifurcation at $\kappa_{r}(0)$, but noise can induce species recovery at a critical point $\kappa_{r}(\varepsilon)$, where $\kappa_{r}(\varepsilon)>\kappa_{r}(0)$. For $\kappa_{r}(0)<\kappa<\kappa_{c}(0)$, the deterministic system has three equilibria: two stable equilibria and an unstable equilibrium in between. The two stable equilibria are two attractors with their own basins of attraction, while the stable manifold of the unstable equilibrium is the basin boundary [102, 12]. Dynamically, the two transition phenomena are the result of noise driving the system across the basin boundary. Transients arise because of the competition between the attractive dynamics in the neighborhoods of the stable equilibria as controlled by the eigenvalues of the Jacobian matrix with negative real part, and stochastic hopping that brings the system out of the attractor [103, 104, 105]. The transient dynamics underlying noise-induced collapse and recovery are the result of stochastic forcing that 


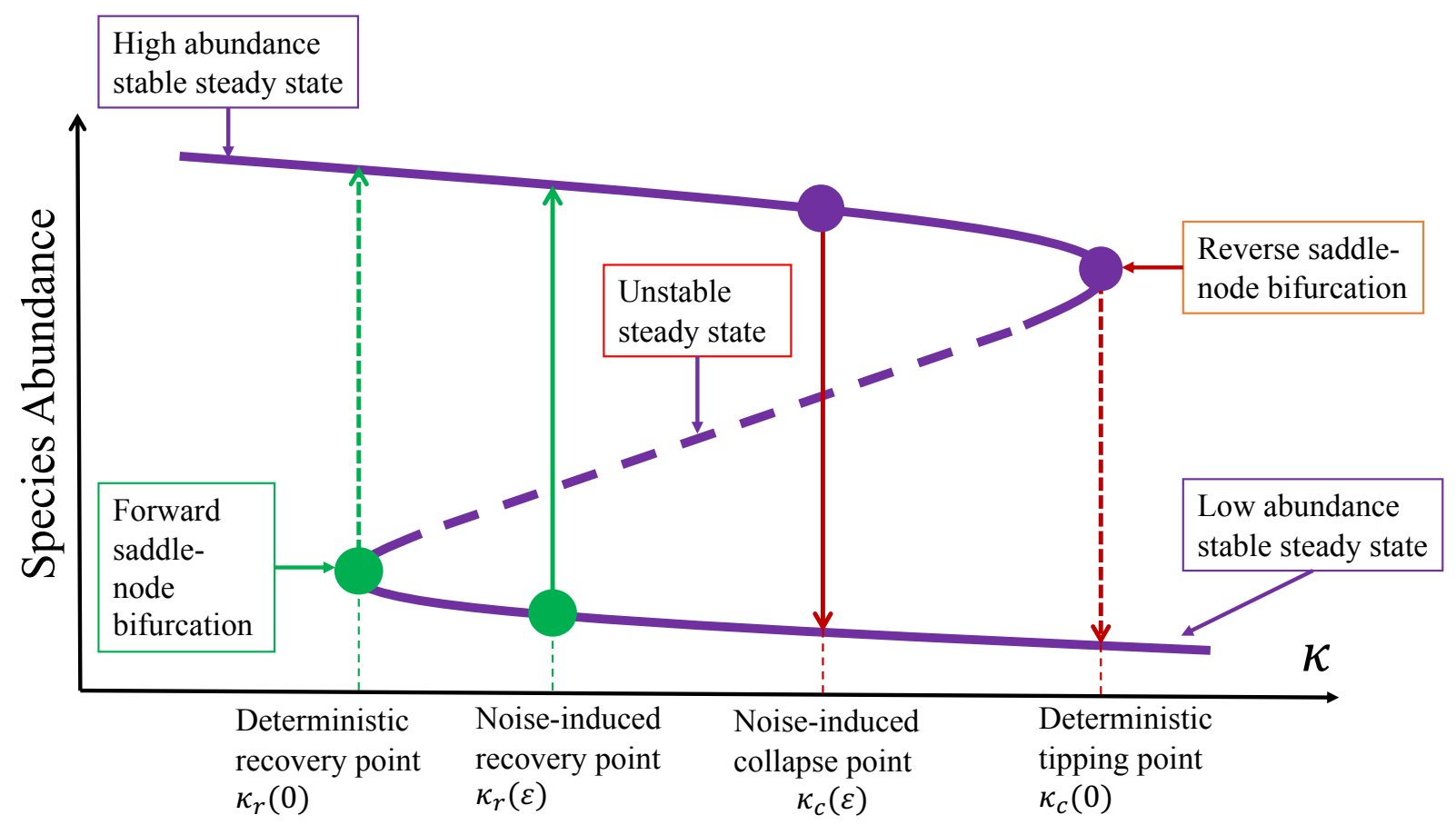

Figure 4: Dynamical mechanism of noise-induced collapse and recovery in mutualistic networks. The bifurcation parameter is the species decay rate $\kappa$. The system has two stable steady states, with high and low abundances, respectively. There is an unstable steady state in between the two stable states. The three equilibrium points are dynamically connected through two saddlenode bifurcations: one corresponding to the tipping point (the reverse one) at $\kappa_{c}(0)$, and another leading to species recovery (the forward one) at $\kappa_{r}(0)$. In the deterministic case, as $\kappa$ increases through $\kappa_{c}(0)$, the system collapses. Under noise of amplitude $\varepsilon$, the collapse can occur earlier at $\kappa_{c}(\varepsilon)$ - the phenomenon of noise-induced collapse. Likewise, as $\kappa$ decreases, noise can induce species recovery at $\kappa_{r}(\varepsilon)$.

drives the system from one stable steady state to another. For an ensemble of trajectories from random initial conditions, the transient time required for the transition is typically exponentially distributed $[106,107]$ and the average transient lifetime $\tau$ depends on the noise amplitude $\varepsilon$. For stronger noise, the transition occurs more quickly, so we expect $\tau$ to decrease with $\varepsilon$. A recent study of four real world mutualistic networks [108] demonstrated the phenomena of noiseinduced collapse and recovery, and confirmed the occurrence of transients. Computation with a two-dimensional reduced model [87] of the empirical networks revealed [108] the following algebraic scaling law between $\tau$ and $\varepsilon$ :

$$
\tau \sim \varepsilon^{-\alpha}
$$

where $\alpha>0$ is the algebraic scaling exponent.

The common dynamical feature of transition from one stable steady state to another between noise-induced collapse and recovery notwithstanding, the specific nature of the noise does play an important role. In particular, environmental noise is independent of the dynamical variables of the system and is thus simply additive, but demographic noise depends on the species abundances. Before reaching the tipping point where the system is in the high-abundance steady state, demographic noise is strong and is the dominant stochastic force to induce a system collapse. In contrast, if the system is in the low-abundance steady state, demographic noise is weak. In this case, the environmental noise becomes the major stochastic driving force for system recovery. The specific roles played by demographic and environmental noises have implications to 
devising strategies to manage high-dimensional ecological systems. For example, because of the detrimental role of demographic noise in causing an ecosystem to collapse, it is imperative to devise methods to reduce the level of demographic noise to keep the system in the healthy state. Conversely, when the system is already close to extinction, a suitable amount of environmental noise may facilitate recovery [89].

\section{$7 \quad$ Flow-kick dynamics and single realizations}

So far, we have focused on systems where the stochastic influence is due to continual noise. But in real ecological systems there may instead be large disturbances at regular or irregular intervals. These exogenous disturbances may be stochastic or, as in some management settings, they may be tightly controlled. In analyses of transients in a deterministic setting [8,9], the focus is often on response to a single perturbation of a system away from its asymptotic state. These ideas provide the background behind the approach we use to deal with cases of repeated large disturbances.

Consider a population that - in the absence of noise - has an attracting state. If the system is subject to recurring disturbance by disease, weather extremes, management, etc., it may never even get close to the asymptotic dynamics, but instead will stabilize in a region of state space where the short-term transient dynamics balance the disturbance.

A familiar example is given by fishery management: an undisturbed fish stock might grow to carrying capacity but, when subject to repeated harvesting, does not recover to full carrying capacity between harvests. Indeed, a management strategy typically maintains stock population significantly below carrying capacity to ensure a high recruitment rate and corresponding yield. Alternatively, in the context of an invasive species, the disturbance pattern might represent a culling strategy. In this case a management strategy of regular removal may be designed to keep the invasive below a threshold way below carrying capacity.

One approach to exploring this phenomenon mathematically is to combine the growth dynamics of the ecosystem with the disturbance dynamic to define a new system whose asymptotic dynamics represent a balance between growth and disturbance. For example, given a continuous population model $\frac{d x}{d t}=f(x)$ that is repeatedly disturbed by a discrete kick $\kappa$ to the state variable, the associated flow-kick system (a special case of impulsive differential equations), is defined by the discrete system

$$
x_{i+1}=x_{i}\left(\tau_{i}\right)+\kappa_{i}
$$

for additive disturbance, or

$$
x_{i+1}=\kappa_{i} x_{i}\left(\tau_{i}\right),
$$

for multiplicative disturbance. Here $x(t)$ is the solution to the undisturbed system with initial condition $x$, and the $i$ th kick $\kappa_{i}$ occurs at time $\tau_{i}$ after the previous kick $\kappa_{i-1}$. In the fishery context, $f(x)$ represents the recruitment function, and the disturbance pattern $\kappa_{i}, \tau_{i}$ represents a harvesting strategy. This framework is used in [57] to quantify resilience of ecosystems to regular recurrent disturbances, and in [53] to study the resilience of socially valued properties of natural systems to recurrent disturbance.

This kind of flow-kick system illustrates the essential role played by transient dynamics in the presence of disturbance to yield different qualitative dynamics, in which kicks can move the asymptotic state of the system in any arbitrary way [53]. More formally, given any point $x^{*}$ in $n$-dimensional state space, and any disturbance time $\tau$, there is a kick $\kappa$ so that $x^{*}$ is an equilibrium of the flow-kick system (3) with $\kappa_{i}=\kappa$ and $\tau_{i}=\tau$ for all $i$. In other words, using a perfectly regular disturbance pattern of fixed kicks at fixed time intervals, one can stabilize the 
disturbed system anywhere in state space, regardless of the location of attracting sets or basins of attraction of the underlying growth dynamics $\frac{d x}{d t}=f(x)$. This idea can be a very powerful conceptual tool in the management of ecosystems [109].

\section{Conclusions}

An overarching challenge in understanding the dynamics of ecological systems is to provide insights on ecologically-realistic time scales in the presence of both environmental variability and stochasticity driven by small population sizes. In this setting, the asymptotic behavior of deterministic systems is not relevant, and instead a focus on transient dynamics is required. Examples of transient behavior have been observed in a variety of ecological systems as previously summarized [8], but a more systematic approach is important for understanding the role of stochasticity in ecological transients, especially in cases where detailed information about the system may be limited. The importance of transients in stochastic systems shows up in a variety of ecological contexts [10] and our contribution here emphasizes both the importance of this phenomenon and the idea that a careful mathematical treatment can find order in what may seem like a series of idiosyncratic examples. This is an area where despite the advances we have covered here, much more work is needed.

The work summarized here can also be thought of as an extension to stochastic variation of the insights that come from studying seasonal dynamics [52]. Analyses of seasonal systems have emphasized ecological implications, such as long-term variation of densities and species succession of plankton communities of temperate lakes across the warm season [110], where the random starting conditions play an important role. The existence of transients with time scales much larger than the time of a single season can guarantee the coexistence of many plankton species within a short time period - which would be impossible for a longer period in a constant environment - since the ecosystem is 're-set' each year in a random fashion [111]. In other words, this type of ecological transient seems to be a robust phenomenon; however, mechanisms of observed long transients in many such systems are still unclear due to the high complexity of communities containing dozens of interacting species, the existence of several time scales, and stochastic aspects. Further work based on the ideas we have developed here will shed light on the ecological implications of stochastic transients [10].

Here, we have emphasized using modeled stochastic dynamics as a way to understand and predict real-world dynamics. We might also consider the inverse problem, in which we could think of stochasticity as obscuring the signal of a processes of interest. As a result, it is tempting to feel that ecological insights would be improved if we could study ecological systems in isolation from stochastic noise. However, if we could observe ecological dynamics in the absence of noise, we would see one behavior - the steady state. A system at or near its equilibrium would simply sit at equilibrium. If our observations began with the system out of equilibrium, we could see a short transient or part of a long transient eventually approaching the equilibrium. In the presence of noise, however, we have the opportunity to see all of these things within a reasonable observation window, as perturbations push the system from one domain to another [112].

One key example that we have highlighted in this work is unexpected shifts between alternative stable states in ecological systems [41]. Much attention has been focused on single shifts, but many systems move more than once between different states. Important quantities, like the expected interval between shifts and the proportion of time the system is expected to be in each state, can be computed with knowledge of each stable state's basin of attraction [113] and the characteristics of the noise. A system that mostly sits at or very near one equilibrium gives us virtually no information about these basins. We may not even know whether other stable states 
exist in such a system. In contrast, a system that experiences enough stochasticity could shift many times [65].

In conclusion, here we argue that an investigation of noisy nonlinear systems reveals a much richer view of the underlying deterministic structure of ecological systems than does focusing exclusively on unperturbed systems. When we observe a system at equilibrium, we can only infer that the equilibrium exists, not what causes it. When we observe how different parts of the system - such as the population densities of different interacting species - change in response to being in different states or configurations, we gain valuable information about the nonlinearities and feedbacks that are present. Extracting these insights, however, requires a good understanding of how the types of stochasticity present interact with these nonlinearities and feedbacks.

Finally, we emphasize that although we have focused on ecological issues and models here, these themes arise in other areas as well. In particular, interactions between stochasticity and transients are clearly important in neuroscience [114, 115], as well as other areas of biology, engineering [116], physics [117] and climate.

\section{Acknowledgements}

This work was conducted as part of the Long Transients and Ecological Forecasting Working Group at the National Institute for Mathematical and Biological Synthesis, supported by the National Science Foundation through NSF Award DBI-1300426, with additional support from The University of Tennessee, Knoxville, and NSF Award no. CCS-1521672. The long term support of NIMBioS directors Lou Gross and Sergey Gavrilets is greatly appreciated. The work of AH was also supported by NSF Grant EF-2025235, and of MLZ by NSF Grant CCF1522054.

\section{References}

[1] Anderson SC, Branch TA, Cooper AB, Dulvy NK. 2017 Black-swan events in animal populations. Proceedings of the National Academy of Sciences 114, 3252-3257.

[2] Anderson SC, Ward EJ. 2019 Black swans in space: modeling spatiotemporal processes with extremes. Ecology 100, e02403.

[3] Hastings A, Higgins K. 1994 Persistence of transients in spatially structured ecological models. Science 263, 1133-1136.

[4] Hastings A. 2001 Transient dynamics and persistence of ecological systems. Ecol. Lett. 4, $215-220$.

[5] Dhamala M, Lai YC, Holt RD. 2001 How often are chaotic transients in spatially extended ecological systems?. Phys. Lett. A 280, 297-302.

[6] Hastings A. 2004 Transients: the key to long-term ecological understanding?. Trends Ecol. Evol. 19, 39-45.

[7] Hastings A. 2016 Timescales and the management of ecological systems. Proc. Nat. Acad. Sci. (USA) 113, 14568-14573.

[8] Hastings A, Abbott KC, Cuddington K, Francis T, Gellner G, Lai YC, Morozov A, Petrivskii S, Scranton K, Zeeman ML. 2018 Transient phenomena in ecology. Science 361, eaat6412. 
[9] Morozov A, Abbott KC, Cuddington K, Francis T, Gellner G, Hastings A, Lai YC, Petrovskii S, Scranton K, Zeeman ML. 2020 Long transients in ecology: Theory and applications. Phys. Life Rev. 32, 1-40.

[10] Shoemaker LG, Sullivan LL, Donohue I, Cabral JS, Williams RJ, Mayfield MM, Chase JM, Chu C, Harpole WS, Huth A, HilleRisLambers J, James ARM, Kraft NJB, May F, Muthukrishnan R, Satterlee S, Taubert F, Wang X, Wiegand T, Yang Q, Abbott KC. 2020 Integrating the underlying structure of stochasticity into community ecology. Ecology 101, $\mathrm{e} 02922$.

[11] Grebogi C, Ott E, Yorke JA. 1983 Crises, sudden changes in chaotic attractors and chaotic transients. Physica D 7, 181-200.

[12] Lai YC, Tél T. 2011 Transient Chaos - Complex Dynamics on Finite-Time Scales. New York: Springer first edition.

[13] Scheffer M, Straile D, van Nes EH, Hosper H. 2001 Climatic warming causes regime shifts in lake food webs. Limnology and Oceanography 46, 1780-1783.

[14] Carpenter SR, Cole JJ, Pace ML, Batt R, Brock WA, Cline T, Coloso J, Hodgson JR, Kitchell JF, Seekell DA, Smith L, Weidel B. 2011 Early Warnings of Regime Shifts: A Whole-Ecosystem Experiment. Science 332, 1079-1082.

[15] Boettiger C, Hastings A. 2012 Quantifying limits to detection of early warning for critical transitions. J. R. Soc. Interface 9, 2527-2539.

[16] Roughgarden J. 1975 A simple model for population dynamics in stochastic environments. Ame. Naturalist 109, 713-736.

[17] Lande R. 1993 Risks of population extinction from demographic and environmental stochasticity and random catastrophes. Ame. Naturalist 142, 911-927.

[18] Yao Q, Tong H. 1994 On prediction and chaos in stochastic systems. Philos. Trans. R. Soc. Lond. A 348, 357-369.

[19] Ludwig D. 1996 The distribution of population survival times. Ame. Naturalist 147, 506526.

[20] Ripa J, Lundberg P, Kaitala V. 1998 A General Theory of Environmental Noise in Ecological Food Webs. Ame. Naturalist 151, 256-263.

[21] Lande R. 1998 Demographic stochasticity and Allee effect on a scale with isotropic noise. Oikos 83, 353-358.

[22] Dennis B. 2002 Allee effects in stochastic populations. Oikos 96, 389-401.

[23] Bonsall MB, Hastings A. 2004 Demographic and environmental stochasticity in predatorprey metapopulation dynamics. J. Animal Ecol. 73, 1043-1055.

[24] Ellner SP, Turchin P. 2005 When can noise induce chaos and why does it matter: A critique. Oikos 111, 620-631.

[25] Lai YC, Liu YR. 2005 Noise Promotes Species Diversity in Nature. Phys. Rev. Lett. 94, 038102 .

[26] Lai YC. 2005 Beneficial role of noise in promoting species diversity through stochastic resonance. Phys. Rev. E 72, 042901. 
[27] Guttal V, Jayaprakash C. 2007 Impact of noise on bistable ecological systems. Ecol. Model. 201, 420-428.

[28] Doney SC, Sailley SF. 2013 When an ecological regime shift is really just stochastic noise. Proc. Nat. Acad. Sci. (USA) 110, 2438-2439.

[29] Bjornstad ON. 2015 Nonlinearity and chaos in ecological dynamics revisited. Proc. Nat. Acad. Sci. (USA) 112, 6252-6253.

[30] O'Regan SM. 2018 How noise and coupling influence leading indicators of population extinction in a spatially extended ecological system. J. Biol. Dyn. 12, 211-241.

[31] Higgins K, Hastings A, Sarvela J, Botsford L. 1997 Stochastic dynamics and deterministic skeletons: population behavior of Dungeness crab. Science 276, 1431-1435.

[32] Dennis B, Desharnais RA, Cushing J, Henson SM, Costantino R. 2003 Can noise induce chaos?. Oikos 102, 329-339.

[33] Heino M. 1998 Noise colour, synchrony and extinctions in spatially structured populations. Oikos 83, 368-375.

[34] Benton TG, Lapsley C, Beckerman AP. 2002 The population response to environmental noise: population size, variance and correlation in an experimental system. J. Animal Ecol. 71, 320-332.

[35] Grenfell BT, Bjornstad ON, Finkenstädt BF. 2002 Dynamics of measles epidemics: scaling noise, determinism, and predictability with the TSIR model. Ecol. Monographs 72, 185-202.

[36] Martín PV, Bonachela JA, Levin SA, Muñoz MA. 2015 Eluding catastrophic shifts. Proc. Nat. Acad. Sci. (USA) 112, E1828-E1836.

[37] Constable GWA, Rogers T, McKane AJ, Tarnita CE. 2016 Demographic noise can reverse the direction of deterministic selection. Proc. Nat. Acad. Sci. (USA) 113, E4745-E4754.

[38] Botsford LW, White JW, Hastings A. 2019 Population dynamics for conservation. Oxford University Press.

[39] Nisbet RM, Gurney W. 2003 Modelling fluctuating populations. Wiley \& Sons.

[40] Nisbet R, Gurney W. 1976 A simple mechanism for population cycles. Nature 263, 319-320.

[41] Scheffer M, Bascompte J, Brock WA, Brovkin V, Carpenter SR, Dakos V, Held H, Van Nes EH, Rietkerk M, Sugihara G. 2009 Early-warning signals for critical transitions. Nature 461, 53-59.

[42] Scheffer M. 2010 Complex systems: foreseeing tipping points. Nature 467, 411-412.

[43] Coulson T, Rohani P, Pascual M. 2004 Skeletons, noise and population growth: the end of an old debate?. Trends in Ecology 8 Evolution 19, 359-364.

[44] Nguyen HT, Rohani P. 2008 Noise, nonlinearity and seasonality: the epidemics of whooping cough revisited. Journal of The Royal Society Interface 5, 403-413.

[45] Alonso D, McKane AJ, Pascual M. 2007 Stochastic amplification in epidemics. Journal of the Royal Society Interface 4, 575-582. 
[46] Király A, Bartos I, Jánosi IM. 2006 Correlation properties of daily temperature anomalies over land. Tellus A: Dynamic Meteorology and Oceanography 58, 593-600.

[47] Pelletier JD. 1997 Analysis and modeling of the natural variability of climate. Journal of Climate 10, 1331-1342.

[48] Lenton TM, Dakos V, Bathiany S, Scheffer M. 2017 Observed trends in the magnitude and persistence of monthly temperature variability. Scientific reports $\mathbf{7}, 1-10$.

[49] Di Cecco GJ, Gouhier TC. 2018 Increased spatial and temporal autocorrelation of temperature under climate change. Scientific reports 8, 1-9.

[50] Bunde A, Eichner JF, Kantelhardt JW, Havlin S. 2005 Long-term memory: A natural mechanism for the clustering of extreme events and anomalous residual times in climate records. Physical Review Letters 94, 048701.

[51] Vesipa R, Ridolfi L. 2017 Impact of seasonal forcing on reactive ecological systems. Journal of Theoretical Biology 419, 23-35.

[52] White ER, Hastings A. 2020 Seasonality in ecology: Progress and prospects in theory. Ecological Complexity 44, 100867.

[53] Zeeman ML, Meyer K, Bussmann E, Hoyer-Leitzel A, Iams S, Klasky IJ, Lee V, Ligtenberg S. 2018 Resilience of socially valued properties of natural systems to repeated disturbance: A framework to support value-laden management decisions. Natural Resource Modeling 31, e12170.

[54] Benzi R, Sutera A, Vulpiani A. 1981 The mechanism of stochastic resonance. Journal of Physics A 14, L453-L457.

[55] B. Lindner B, Garcia-Ojalvo J, Neiman A, Schimansky-Geier L. 2004 Effects of noise in excitable systems. Physics Reports 392, 321-424.

[56] Moskalenko OI, Koronovskii AA, Zhuravlev MO, Hramov AE. 2018 Characteristics of noiseinduced intermittency. Chaos, Solitons \& Fractals 117, 269-275.

[57] Meyer K, Hoyer-Leitzel A, Iams S, Klasky I, Lee V, Ligtenberg S, Bussmann E, Zeeman ML. 2018 Quantifying resilience to recurrent ecosystem disturbances using flow-kick dynamics. Nature Sustainability 1, 671-678.

[58] Scheffer M, Carpenter S, Foley J, Folke C, Walker B. 2001 Catastrophic shifts in ecosystems. Nature 413, 591-596.

[59] Taylor KC, Lamorey G, Doyle G, Alley RB, Grootes P, Mayewski PA, White J, Barlow L. 1993 The 'flickering switch'of late Pleistocene climate change. Nature 361, 432-436.

[60] Wang R, Dearing JA, Langdon PG, Zhang E, Yang X, Dakos V, Scheffer M. 2012 Flickering gives early warning signals of a critical transition to a eutrophic lake state. Nature 492, $419-422$.

[61] Clements CF, Ozgul A. 2016 Including trait-based early warning signals helps predict population collapse. Nature communications $\mathbf{7}, 1-8$.

[62] Keeling MJ, Rohani P, Grenfell BT. 2001 Seasonally forced disease dynamics explored as switching between attractors. Physica D: Nonlinear Phenomena 148, 317-335. 
[63] Fiasconaro A, Spagnolo B, Boccaletti S. 2005 Signatures of noise-enhanced stability in metastable states. Physical Review E 72, 061110.

[64] Spalding C, Doering CR, Flierl GR. 2017 Resonant activation of population extinctions. Physical Review E 96, 042411.

[65] Abbott KC, Dakos V. in press Mapping the distinct origins of bimodality in a classic model with alternative stable states. Theoretical Ecology.

[66] Morozov AY, Petrovskii SV. 2009 Excitable population dynamics, biological control failure, and spatiotemporal pattern formation in a model ecosystem. Bull. Math. Biol. 71, 863-887.

[67] Borowski P, Kuske R, Li YX, Cabrera JL. 2010 Characterizing mixed mode oscillations shaped by noise and bifurcation structure. Chaos 20, 043117.1-043117.22.

[68] Desroches M, Guckenheimer J, Krauskopf B, Kuehn C, Osinga H, Wechselberger M. 2012 Mixed-mode oscillations with multiple time scales. Siam Review 54, 211-288.

[69] Toral R, Mirasso CR, Hernández-Garcıa E, Piro O. 2001 Analytical and numerical studies of noise-induced synchronization of chaotic systems. Chaos: An Interdisciplinary Journal of Nonlinear Science 11, 665-673.

[70] Lyles D et al.. 2009 The role of large environmental noise in masting: general model and example from pistachio trees. J. Theor. Biol. 253, 701-713.

[71] Rosenstock T, Hastings A, Koenig W, Lyles D, Brown P. 2011 Testing Moran's theorem in an agroecosystem. Oikos 120, 1434-1440.

[72] Moskalenko O, Koronovskii A, Shurygina S. 2011 Intermittent behavior at the boundary of noise-induced synchronization. Technical Physics 56, 1369.

[73] Renshaw E. 1991 Modelling biological populations in space and time. Cambridge, UK: Cambridge University Press.

[74] Gammaitoni L, Hänggi P, Jung P, Marchesoni F. 2009 Stochastic Resonance: A remarkable idea that changed our perception of noise. Eur. Phys. J. B 69, 1-3.

[75] Bjørnstad ON, Nisbet RM, FROMENTIN JM. 2004 Trends and cohort resonant effects in age-structured populations. Journal of animal ecology 73, 1157-1167.

[76] Grebogi C, Ott E, Yorke JA. 1983 Crises, sudden changes in chaotic attractors, and transient chaos. Physica D: Nonlinear Phenomena 7, 181-200.

[77] Hastings A, Powell T. 1991 Chaos in a three-species food chain. Ecology 72, 896-903.

[78] Do Y, Lai YC. 2004 Extraordinarily superpersistent chaotic transients. Europhys. Lett. 67, 914-920.

[79] Do Y, Lai YC. 2005 Scaling laws for noise-induced superpersistent chaotic transients. Phys. Rev. E 71, 046208.

[80] Bascompte J, Jordano P, Melián CJ, Olesen JM. 2003 The nested assembly of plant-animal mutualistic networks. Proc. Natl. Acad. Sci. (USA) 100, 9383-9387.

[81] Guimaraes PR, Jordano P, Thompson JN. 2011 Evolution and coevolution in mutualistic networks. Ecol. Lett. 14, 877-885. 
[82] Nuismer SL, Jordano P, Bascompte J. 2013 Coevolution and the architecture of mutualistic networks. Evolution 67, 338-354.

[83] Lever JJ, Nes EH, Scheffer M, Bascompte J. 2014 The sudden collapse of pollinator communities. Ecol. Lett. 17, 350-359.

[84] Rohr RP, Saavedra S, Bascompte J. 2014 On the structural stability of mutualistic systems. Science 345, 1253497.

[85] Dakos V, Bascompte J. 2014 Critical slowing down as early warning for the onset of collapse in mutualistic communities. Proc. Natl. Acad. Sci. (USA) 111, 17546-17551.

[86] Guimaraes PR, Pires MM, Jordano P, Bascompte J, Thompson JN. 2017 Indirect effects drive coevolution in mutualistic networks. Nature 550, 511-514.

[87] Jiang J, Huang ZG, Seager TP, Lin W, Grebogi C, Hastings A, Lai YC. 2018 Predicting tipping points in mutualistic networks through dimension reduction. Proc. Natl. Acad. Sci. (USA) 115, E639-E647.

[88] Jiang J, Hastings A, Lai YC. 2019 Harnessing tipping points in complex ecological networks. J. R. Soc. Interface 16, 20190345.

[89] Meng Y, Jiang J, Grebogi C, Lai YC. 2020 Noise-enabled species recovery in the aftermath of a tipping point. Phys. Rev. E 101, 012206.

[90] Ohgushi T, Schmitz O, Holt RD. 2012 Trait-Mediated Indirect Interactions: Ecological and Evolutionary Perspectives. Cambridge UK: Cambridge Univ. Press.

[91] Scheffer M. 2004 Ecology of Shallow Lakes. Springer Science \& Business Media.

[92] Wysham DB, Hastings A. 2010 Regime shifts in ecological systems can occur with no warning. Ecol. Lett. 13, 464-472.

[93] Drake JM, Griffen BD. 2010 Early warning signals of extinction in deteriorating environments. Nature 467, 456-459.

[94] Dai L, Vorselen D, Korolev KS, Gore J. 2012 Generic indicators for loss of resilience before a tipping point leading to population collapse. Science 336, 1175-1177.

[95] Ashwin P, Wieczorek S, Vitolo R, Cox P. 2012 Tipping points in open systems: bifurcation, noise-induced and rate-dependent examples in the climate system. Phil. Trans. Roy. Soc. A 370, 1166-1184.

[96] Lenton TM, Livina VN, Dakos V, van Nes EH, Scheffer M. 2012 Early warning of climate tipping points from critical slowing down: comparing methods to improve robustness. Phil. Trans. Roy. Soc. A 370, 1185-1204.

[97] Barnosky AD, Hadly EA, Bascompte J, Brown ELBJH, Fortelius M, Getz WM, Harte J, Hastings A, Marquet PA, Martinez ND, Mooers A, Roopnarine P, Vermeij G, Williams JW, Gillespie R, Kitzes J, Marshall C, Matzke N, Mindell DP, Revilla E, Smith AB. 2012 Approaching a state shift in Earth's biosphere. Nature 486, 52-58.

[98] Boettiger C, Hastings A. 2013 Tipping points: From patterns to predictions. Nature 493, $157-158$.

[99] Tylianakis JM, Coux C. 2014 Tipping points in ecological networks. Trends. Plant. Sci. 19, 281-283. 
[100] Lontzek TS, Cai YY, Judd KL, Lenton TM. 2015 Stochastic integrated assessment of climate tipping points indicates the need for strict climate policy. Nat. Clim. Change 5, $441-444$.

[101] Gualdia S, Tarziaa M, Zamponic F, Bouchaudd JP. 2015 Tipping points in macroeconomic agent-based models. J. Econ. Dyn. Contr. 50, 29-61.

[102] McDonald SW, Grebogi C, Ott E, Yorke JA. 1985 Fractal basin boundaries. Physica D 17, 125-153.

[103] Poon L, Grebogi C. 1995 Controlling Complexity. Phys. Rev. Lett. 75, 4023-4026.

[104] Kraut S, Feudel U, Grebogi C. 1999 Preference of attractors in noisy multistable systems. Phys. Rev. E 59, 5253-5260.

[105] Liu Z, Lai YC, Billings L, Schwartz IB. 2002 Transition to Chaos in Continuous-Time Random Dynamical Systems. Phys. Rev. Lett. 88, 124101.

[106] Grebogi C, Ott E, Romeiras F, Yorke JA. 1987 Critical exponents for crisis-induced intermittency. Phys. Rev. A 36, 5365-5380.

[107] Sommerer JC, Ott E, Grebogi C. 1991 Scaling law for characteristic times of noise-induced crises. Phys. Rev. A 43, 1754.

[108] Meng Y, Lai YC, Grebogi C. 2020 Tipping point and noise-induced transients in ecological networks. Journal of the Royal Society Interface 17, 20200645.

[109] Francis TB, Abbott KC, Cuddington K, Gellner G, Hastings A, Lai YC, Morozov A, Petrovskii S, Zeeman ML. 2021 Management implications of long transients in ecological systems. Nature Ecology \& Evolution pp. 1-10.

[110] Jager C, Diehl S, Matauschek C, Klausmeier C, Stibor H. 2008 Transient dynamics of pelagic producer grazer systems in a gradient of nutrients and mixing depths. Ecology 89, $1272-1286$.

[111] Huisman J, Weissing FJ. 1999 Biodiversity of plankton by species oscillations and chaos. Nature 402, 407-410.

[112] Boettiger C. 2018 From noise to knowledge: how randomness generates novel phenomena and reveals information. Ecology letters 21, 1255-1267.

[113] Nolting BC, Abbott KC. 2016 Balls, cups, and quasi-potentials: quantifying stability in stochastic systems. Ecology 97, 850-864.

[114] Rabinovich M, Huerta R, Laurent G. 2008 Transient Dynamics for Neural Processing. Science 321, 48-50.

[115] Palmigiano A, Geisel T, Wolf F, Battaglia D. 2017 Flexible information routing by transient synchrony. Nature neuroscience 20, 1014.

[116] Masri SF, Caffrey JP. 2017 Transient response of a SDOF system with an inerter to nonstationary stochastic excitation. Journal of Applied Mechanics $\mathbf{8 4}$.

[117] Šiler M, Ornigotti L, Brzobohatỳ O, Jákl P, Ryabov A, Holubec V, Zemánek P, Filip R. 2018 Diffusing up the hill: Dynamics and equipartition in highly unstable systems. Physical review letters 121, 230601. 